\title{
Vascular calcification in skin and subcutaneous tissue in patients with chronic and end-stage kidney disease
}

Irene Ruderman ${ }^{1,2^{*}}$, Tim D. Hewitson ${ }^{1,2}$, Edward R. Smith ${ }^{1,2}$, Stephen G. Holt ${ }^{1,2}$, Belinda Wigg ${ }^{1}$ and Nigel D. Toussaint ${ }^{1,2}$

\begin{abstract}
Background: Vascular calcification (VC) is well described in large- and medium-sized vessels in patients with chronic kidney disease (CKD), especially in those with end-stage kidney disease (ESKD) on dialysis. Medial calcification is particularly prevalent in this population and contributes to arterial stiffness and increased cardiovascular mortality and morbidity. Apart from in the setting of calciphylaxis, few studies have assessed skin and subcutaneous calcification and associations with abnormalities of bone and mineral metabolism in patients with CKD.

Methods: We performed a single-centre observational study to evaluate incisional skin tissue samples from three anatomical sites in patients with different stages of CKD undergoing elective surgery. We compared these samples to skin samples of a control cohort without CKD. Staining for calcification was performed with von Kossa method. A subgroup of skin samples were assessed by RT-PCR for upregulation of pro-calcific gene transcripts for tissue nonspecific alkaline phosphatase (TNAP) and Runt-related transcription factor 2 (RUNX2).

Results: Forty-five patients were evaluated, 34 with CKD (including ESKD) and 11 control patients. VC was identified in 15 skin samples (13 CKD/ESKD and 2 controls). VC was present in the dermal and subcutaneous tissues of the neck, abdomen and arm samples. Two different histological types of VC were identified: speckled medial calcification and internal elastic lamina calcification. Presence of perieccrine calcification was identified in 14 samples, 10 with concurrent VC. There were no significant differences in serum parathyroid hormone, phosphate or calcium in patients with or without VC. Expression of TNAP or RUNX2 was not increased in samples from patients with ESKD or those with histological evidence of calcification.
\end{abstract}

Conclusion: This study reports the novel finding of dermal and subcutaneous calcification in multiple anatomical locations in 38\% of patients with advanced CKD/ESKD undergoing elective surgery but free from calciphylaxis.

Keywords: Vascular calcification, Chronic kidney disease, End-stage kidney disease, Calcific uraemic arteriolopathy

\footnotetext{
* Correspondence: irene_ruderman@hotmail.com

'Department of Nephrology, The Royal Melbourne Hospital, 300 Grattan St, Parkville, Victoria 3050, Australia

2Department of Medicine (RMH), The University of Melbourne, Parkville,

Victoria, Australia
}

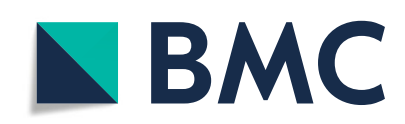

(c) The Author(s). 2020 Open Access This article is licensed under a Creative Commons Attribution 4.0 International License, which permits use, sharing, adaptation, distribution and reproduction in any medium or format, as long as you give appropriate credit to the original author(s) and the source, provide a link to the Creative Commons licence, and indicate if changes were made. The images or other third party material in this article are included in the article's Creative Commons licence, unless indicated otherwise in a credit line to the material. If material is not included in the article's Creative Commons licence and your intended use is not permitted by statutory regulation or exceeds the permitted use, you will need to obtain permission directly from the copyright holder. To view a copy of this licence, visit http://creativecommons.org/licenses/by/4.0/ The Creative Commons Public Domain Dedication waiver (http://creativecommons.org/publicdomain/zero/1.0/) applies to the data made available in this article, unless otherwise stated in a credit line to the data. 


\section{Background}

Vascular calcification (VC) is prevalent and well described in large- and medium-sized vessels of patients with chronic kidney disease (CKD), especially in those with end-stage kidney disease (ESKD) on dialysis [1, 2]. Patients with CKD are at higher risk of VC compared to the general population, irrespective of age and comorbidity burden [3]. Medial calcification is particularly prevalent in the CKD population and is associated with arterial stiffening and higher cardiovascular morbidity and mortality $[4,5]$. Medial arterial calcification has been identified in multiple anatomical locations in patients with ESKD, including normal breast tissue from mastectomy samples [6], as well as subcutaneous tissue of lower limb amputations [7]. In both locations, VC has been identified in vessels in the presence of other patient risk factors for calcification, including active malignancy or peripheral vascular disease.

Conversely, the presence of $\mathrm{VC}$ of small vessels in the skin and subcutaneous tissue has only been systemically described in ESKD patients with calciphylaxis [8], although recent studies suggest even this may not be a specific finding and VC can be present in patients with peripheral vascular disease without the presence of calciphylaxis [7]. Calciphylaxis is considered an accelerated template of widespread VC. It is characterized by severe vessel ischaemia accompanied by vessel thrombosis and surrounding panniculitis [9], ultimately resulting in painful skin lesions which typically break down to nonhealing ulcers that are prone to infection, with accompanying high mortality $[10,11]$. However, calciphylaxis is a rare condition and only occurs in a very small minority of patients with ESKD with approximately 3.5 cases per 1000 patient years in those receiving chronic haemodialysis therapy [12] To date, there is little information about the prevalence of calcification in skin and subcutaneous tissue in patients with advanced CKD and ESKD outside the setting of calciphylaxis, and if present, whether it is related to disordered bone and mineral metabolism.

The aim of the present study was to characterize VC changes in the skin and subcutaneous tissue of patients with CKD compared to a control group. We also aimed to evaluate associations between skin calcification and biochemical markers of deranged bone and mineral metabolism and identify any other histological features present that are typically described in calciphylaxis.

\section{Methods}

\section{Patients}

Between May 2017 and March 2019 patients undergoing a planned surgical procedure performed by the Nephrology Surgical Team at The Royal Melbourne Hospital were approached to participate in this single-centre cross-sectional study. Patients enrolled underwent elective surgical procedures including parathyroidectomy, hernia repair and arteriovenous fistula (AVF) formation. A fasting blood sample was collected at the time of surgical procedure. Patients over the age of 18 and able to provide informed consent were included in the study. There were no exclusion criteria.

\section{Ethics approval and consent to participate}

The study was approved by the Melbourne Health Human Research Ethics Committee (\#HREC 2017.023) and was conducted in accordance with the Declaration of Helsinki. Written informed consent was obtained for this study.

\section{Histology}

A full-thickness incisional skin sample was collected by the operating surgeon from the surgical site. Tissue samples were fixed in neutral buffered formalin and processed to paraffin wax for histology. Serial paraffin embedded skin sections were stained with haematoxylin and eosin $(\mathrm{H} \& \mathrm{E})$ and von Kossa. Cross-sections of skin and subcutaneous tissue were evaluated in their entirety.

Counting of vessels was performed on slides stained with $H \& E$ due to ease of identifying vessels. Each cross section contained epidermal, dermal and subcutaneous tissue, with tissue blocks re-cut if they did not contain all three areas. Vessel size was estimated using a graticule. Typically, arterioles $\leq 50 \mu \mathrm{m}$ in diameter have either absent or partially complete internal elastic lamina (IEL) $[13,14]$. As a result, differentiating terminal arterioles from terminal venules is very challenging, therefore all structures with a lumen were defined as vessels. Samples with greater than 10 vessels per cross section were considered to have adequate vessel number. H\&E sections were examined for evidence of intimal hyperplasia, intimal thrombosis, pannicultis or hyperplasia.

Identification of calcification was performed on slides stained with von Kossa. In brief, sections were dewaxed and rehydrated before being immersed in a solution of freshly prepared 5\% Aqueous Silver Nitrate. Tissue sections were placed at close range under a bench lamp and exposed to a strong light source for $30 \mathrm{~min}$. They were then washed in distilled water. To remove the unreacted silver, slides were covered with $5 \%$ Sodium Thiosulphate for 2 minutes. This was rinsed in distilled water before being counter stained with Working Eosin solution for 2 minutes. For each case, the following features were graded as either present or absent: calcification of any sized vessels, presence of extra-vascular calcification, IEL calcification. Results were expressed as a \% of all vessels in the section. Histologic sections of all cases were counted independently by two investigators (IR and BW) 
with results presented as an average of the two observers. Malignant necrotic breast tissue was used as a positive control for the von Kossa stain.

\section{Real-time reverse transcriptase polymerase chain reaction (RT-PCR)}

Total RNA was isolated from a subgroup (13 out of 43) of patient samples stored in RNAlater solution (Invitrogen, Carlsbad, CA, USA) using the RNEeasy Fibrous Mini Kit (QIAGEN, Hilden, Germany) in accordance with manufacturer's instructions. To minimise sampling error, triplicate $30 \mathrm{mg}$ portions of tissue from each sample were processed. Quantification of RNA was performed using the Qubit 4 Fluorometer and Quant-IT RNA Assay Kit (Thermo Fisher Scientific, Waltham, MA, USA) according to the manufacturer's instruction with $1 \mu \mathrm{g}$ used as a template for cDNA synthesis using the iScript RT supermix kit (BioRad, Hercules, CA, USA). Quantitative real-time PCR (qRT-PCR) was performed in triplicate in a CFX96 cycler (Bio-Rad) using equal volumes of cDNA $(2 \mu \mathrm{L})$ and the SsoAdvanced ${ }^{\mathrm{m}}$ Universal SYBR Green Supermix (BioRad) and validated PCR Prime assay primer pairs (BioRad): TNAP (Unique Assay ID:qHsaCID0010031); Runx2 (Unique Assay ID: qHsaCID006726); and glyceraldehyde3-phosphate dehydrogenase (Gapdh) (Unique Assay ID: qHsaCED0038674). PCR conditions were set according to the manufacturer's instructions. Melt curve analysis was performed to verify the purity and specificity of the amplicons. Threshold cycles were calculated using CFX Manager Software (Bio-Rad). The mRNA level of target genes was normalised to the house keeping gene Gapdh, and expressed relative to an appropriate control using the $2^{\wedge}(-\Delta \Delta C t)$ method. Results are presented as the mean of all three samples.

\section{Statistical analysis}

A sample size estimation for this cross-sectional study was 50 patients based on feasibility with the number of patients undergoing elective surgery at our institution over the recruitment period as well as a larger enough cohort to allow for heterogeneity of skin sample sites and different stages of CKD. All data were summarised, and results reported as mean (standard deviation [SD]) or median (inter-quartile range [IQR]) for continuous data and number (percentage, \%) for categorical variables. Comparison of the values of continuous variables between groups was made using an unpaired t test or Wilcoxon signed-rank test as appropriate. Chi-squared and Fisher's exact tests were used to investigate associations between various categorical variables. Two-tailed $P$ values of $<0.05$ were considered to be significant. All statistical analyses were performed using SPSS version 21.0 for Macintosh (SPPS, Chicago, IL). Graphics were created with GraphPad Prism 8 for Macintosh (la Jolla, CA, USA).

\section{Results}

Demographic and biochemical characteristics

Forty-five patients undergoing elective surgical procedures were enrolled in the study. Eleven patients with normal kidney function (mean eGFR $83 \pm 10 \mathrm{~mL} / \mathrm{min} /$ $1.73 \mathrm{~m}^{2}$ ) were included in the control group. Of these 11, 2 underwent donor nephrectomies, 5 hernia repairs, 1 laparoscopic cholecystectomy and 3 total thyroidectomies. Of the 34 patients with advanced CKD (mean eGFR $21 \pm 0.5 \mathrm{ml} / \mathrm{min} / 1.73 \mathrm{~m}^{2}$ ) and ESKD, 20 underwent AVF creation, superficialization or ligation, 7 parathyroidectomies, 2 kidney transplant nephrectomies, 2 peritoneal dialysis catheter insertions, 2 hernia repairs and 1 total thyroidectomy.

Relevant patient comorbidities and biochemistry are summarised in Table 1. The mean age of the CKD group was $60 \pm 16$ years; $76 \%(n=26)$ of patients were on haemodialysis, $15 \%(n=5)$ on peritoneal dialysis and $9 \%$ $(n=3)$ had CKD stages 4 and 5 not on dialysis. The comorbidity burden was greater in the CKD group

Table 1 Clinical features of study cohort

\begin{tabular}{|c|c|c|c|}
\hline Demographics & CKD $(\boldsymbol{n}=34)$ & $\begin{array}{l}\text { Control } \\
(\boldsymbol{n}=11)\end{array}$ & $\begin{array}{l}\boldsymbol{P} \\
\text { value }\end{array}$ \\
\hline Age, years & $60 \pm 16$ & $62 \pm 9$ & 0.3 \\
\hline Gender, male & $22(69 \%)$ & $4(36 \%)$ & \\
\hline BMI $\left(\mathrm{kg} / \mathrm{m}^{2}\right)$ & 27 [24-33] & $27[24-31]$ & 0.8 \\
\hline Dialysis modality & $\begin{array}{l}26 \mathrm{HD}, 5 \mathrm{PD}, 3 \\
\mathrm{CKD}\end{array}$ & $\mathrm{n} / \mathrm{a}$ & \\
\hline Time on dialysis (years) & $4[2.8-9.8]$ & & \\
\hline \multicolumn{4}{|l|}{ Comorbidities } \\
\hline Smoker (current) & $5(15 \%)$ & $2(18 \%)$ & 0.6 \\
\hline Smoker (former) & $13(38 \%)$ & $2(18 \%)$ & 0.8 \\
\hline Diabetes & $13(38 \%)$ & $2(18 \%)$ & 0.8 \\
\hline Hypertension & $24(70 \%)$ & $2(18 \%)$ & 0.7 \\
\hline $\mathrm{IHD}$ & $7(20 \%)$ & 0 & \\
\hline PVD & $4(12 \%)$ & 0 & \\
\hline Parathyroidectomy & $4(12 \%)$ & 0 & \\
\hline \multicolumn{4}{|l|}{ Biochemical parameters } \\
\hline $\begin{array}{l}\text { Corrected calcium (mmol/ } \\
\text { L) }\end{array}$ & $2.3 \pm 0.2$ & $2.3 \pm 0.2$ & 0.65 \\
\hline Phosphate (mmol/L) & $1.9 \pm 0.6$ & $1.1 \pm 0.2$ & 0.002 \\
\hline ALP (IU/L) & 107 [81-149] & $\begin{array}{l}74[64- \\
102]\end{array}$ & 0.03 \\
\hline PTH (pmol/L) & $62[37-148]$ & $4.6[0.9-8]$ & $\begin{array}{l}< \\
0.001\end{array}$ \\
\hline
\end{tabular}

Results are mean $\pm \mathrm{SD}$, median $[\mathrm{IQR}]$ or number (\%)

Abbreviations: $A L P$ alkaline phosphatase, $B M I$ body mass index, $H D$ haemodialysis, IHD ischaemic heart disease, $P D$ peritoneal dialysis, $P T H$ parathyroid hormone, $P V D$ peripheral vascular disease 
Table 2 Anatomical and histological features of skin tissue samples

\begin{tabular}{lll}
\hline Histologic features & $\begin{array}{l}\text { CKD } \\
(\boldsymbol{n}=34)\end{array}$ & $\begin{array}{l}\text { Control } \\
(\boldsymbol{n}=11)\end{array}$ \\
\hline Location, number of samples & & \\
- Neck & 8 & 3 \\
- Abdomen & 6 & 8 \\
- Arm & 20 & 0 \\
Vessel number, mean \pm SD & & \\
- All sites & $42 \pm 17$ & $34 \pm 13$ \\
- Neck & $37 \pm 7$ & 47 \\
- Abdomen & $63 \pm 18$ & $39 \pm 15$ \\
- Arm & $37 \pm 14$ & $\mathrm{n} / \mathrm{a}$ \\
Vascular calcification present, number (\%) & $13(38 \%)$ & $2(18 \%)$ \\
Perieccrine calcification, number (\%) & $13(38 \%)$ & $1(9 \%)$ \\
\hline
\end{tabular}

Results are mean \pm SD, or number (\%)

compared to the control group; $53 \%(n=18)$ of patients were either former or current smokers, $38 \%(n=13)$ had a history of diabetes, $70 \%(n=24)$ had hypertension, and $12 \%(n=4)$ were documented to have peripheral vascular disease. The median body mass index (BMI) of patients was in the overweight range for both the control and CKD cohort. As expected, there were significant differences in mineral biochemistry between the control and CKD cohort, with higher levels of serum parathyroid hormone $(\mathrm{PTH})(p<0.001)$, phosphate $(p=0.002)$ and alkaline phosphatase (ALP) $(p=0.03)$ in patients with CKD. None of the patients were receiving warfarin. Sixteen patients in the CKD group were prescribed calcitriol and 25 prescribed phosphate binders, 12 of who were on a calcium-based phosphate binder.

\section{Anatomical and histological features of participants}

Anatomical location and histologic features of the skin samples are summarized in Table 2. All sections contained a minimum of 10 vessels, with a mean of $41 \pm 16$ and $34 \pm 17$ vessels per slide in the CKD/ ESKD and control groups, respectively. All sections contained full thickness skin tissue including epidermis, dermis and variable amount of subcutaneous tissue depending on the anatomical location of the sample. The mean number of vessels did not differ significantly across the three anatomical locations $(p=0.17)$ or between groups. Vessels $\leq 50 \mu \mathrm{m}$ in diameter accounted for $75 \%$ of total vessels counted. The largest vessel identified was $500 \mu \mathrm{m}$ in diameter from an arm skin sample. Each sample had a mean of $5 \pm 0.6$ vessels $\geq 100 \mu \mathrm{m}$ in diameter. Figure 1 demonstrates the number of vessels per vessel diameter in each patient based on anatomical location.

\section{CKD/ESKD patients have high rates of VC}

Calcification was observed on von Kossa staining in small (typical diameter 50-100 $\mu \mathrm{m}$ lumen) and mediumsized (typical diameter $>100 \mu \mathrm{m}$ lumen) vessels in 13 CKD/ESKD patients (38\%) (CKD stage $4 n=1$, ESKD $n=12)$ and 2 control patients $(18 \%)$. The difference in proportion of patients with calcification did not differ between CKD/ESKD patients and the control cohort $(p=0.28)$, however this is likely due to the small study sample size. In the majority of samples $(n=8), \mathrm{VC}$ was predominantly localized to subcutaneous tissue, whereas in 5 samples there was an equal distribution of calcified vessels in the dermis and subcutaneous tissue. Irrespective of the tissue layer affected, VC was observed in vessels of all sizes, ranging from 50 to $200 \mu \mathrm{m}$ in diameter. We identified more calcified vessels in neck skin samples of patients undergoing parathyroidectomy than other surgeries, which may reflect the subset of patients with severe secondary hyperparathyroidism. VC was identified at each of the three anatomical sites in patients with CKD/ESKD and in both sites sampled in the controls. Percentage of calcified vessels based on anatomical location is demonstrated in Fig. 2 (vessels $>100 \mu \mathrm{m}$ in diameter identified as small arteries or arterioles) and Fig. 3 (all calcified vessels). The percentage of calcified vessels in this study however likely underestimates total arterial calcification since the count denominator includes veins and venules.

\section{VC was seen in vessel media and internal elastic lamina}

To better understand the nature of $\mathrm{VC}$, we further evaluated its histological appearance and location within the vessel and whether it was granular, confluent or circumferential in nature. $\mathrm{VC}$ of the media was seen in patients with CKD/ESKD, with no evidence of intimal calcification. Medial VC was present as circumferential and granular calcifications in small vessels, although we were unable to determine if this involved the IEL (Fig. 4a). In larger vessels the granular calcification was noted within the vessel media and alongside the IEL (Fig. 4b), and some vessels displayed confluent areas of calcification (Fig. 4c). There was no evidence of circumferential medial calcification seen, however there was evidence of circumferential linear calcification of the IEL affecting vessels $>100 \mu \mathrm{m}$ in diameter in two patients with ESKD (Fig. 4d).

\section{Concurrence of perieccrine calcification in samples with medial VC}

Extra-vascular calcification was identified surrounding sweat glands (perieccrine) in 13 participants with CKD and one control participant (Fig. 4e). Of the participants with perieccrine calcification, 10 also had VC. There was no intimal hyperplasia or other pathological features 

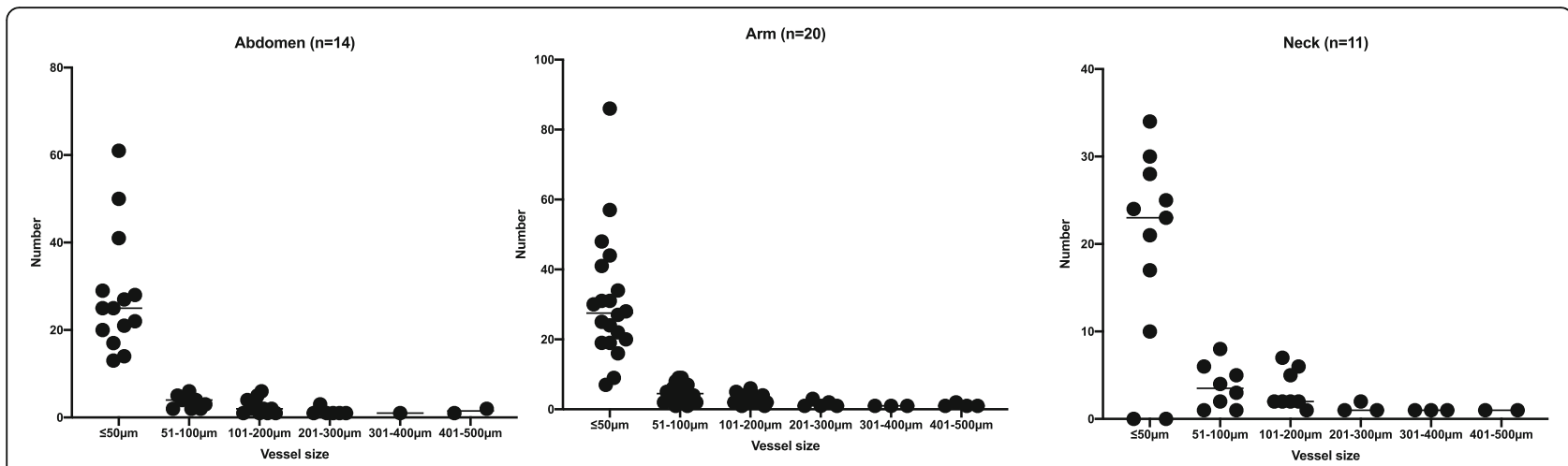

Fig. 1 Number of vessels per vessel diameter in each patient based on anatomical location. Each dot per column represent individual patient

consistent with calciphylaxis, including intimal thrombosis, panniculitis or hyperplasia. No other form of extra-vascular calcification was identified, including subcutaneous septa.

VC in the control group was mild compared to the CKD/ ESKD group

Two patients in the control group had presence of subtle and stippled medial calcification (Fig. 4f). Both patients were female, aged 65 and 71 years of age with no significant co-morbidities and normal biochemistry. One patient underwent a donor nephrectomy and had calcification present in $11 \%$ of vessels, mostly involving large arterioles. The second patient underwent a total thyroidectomy and was found to have calcification in
$20 \%$ of vessels, all within subcutaneous tissue. The pattern of $\mathrm{VC}$ in the control group appeared distinct to that in the CKD/ESKD group with no evidence of granular or confluent calcification, or involvement of the IEL. There was evidence of perieccrine calcification in one patient in the control cohort.

Similar demographic and biochemical markers of mineral metabolism in patients with and without skin and subcutaneous VC

In order to determine if the presence of $\mathrm{VC}$ was related to demographic and clinical features, we compared the specific characteristics of CKD/ESKD patients with and without VC (Table 3). Patients in both groups were of a similar age $(p=0.6)$ and dialysis vintage $(p=0.2)$.

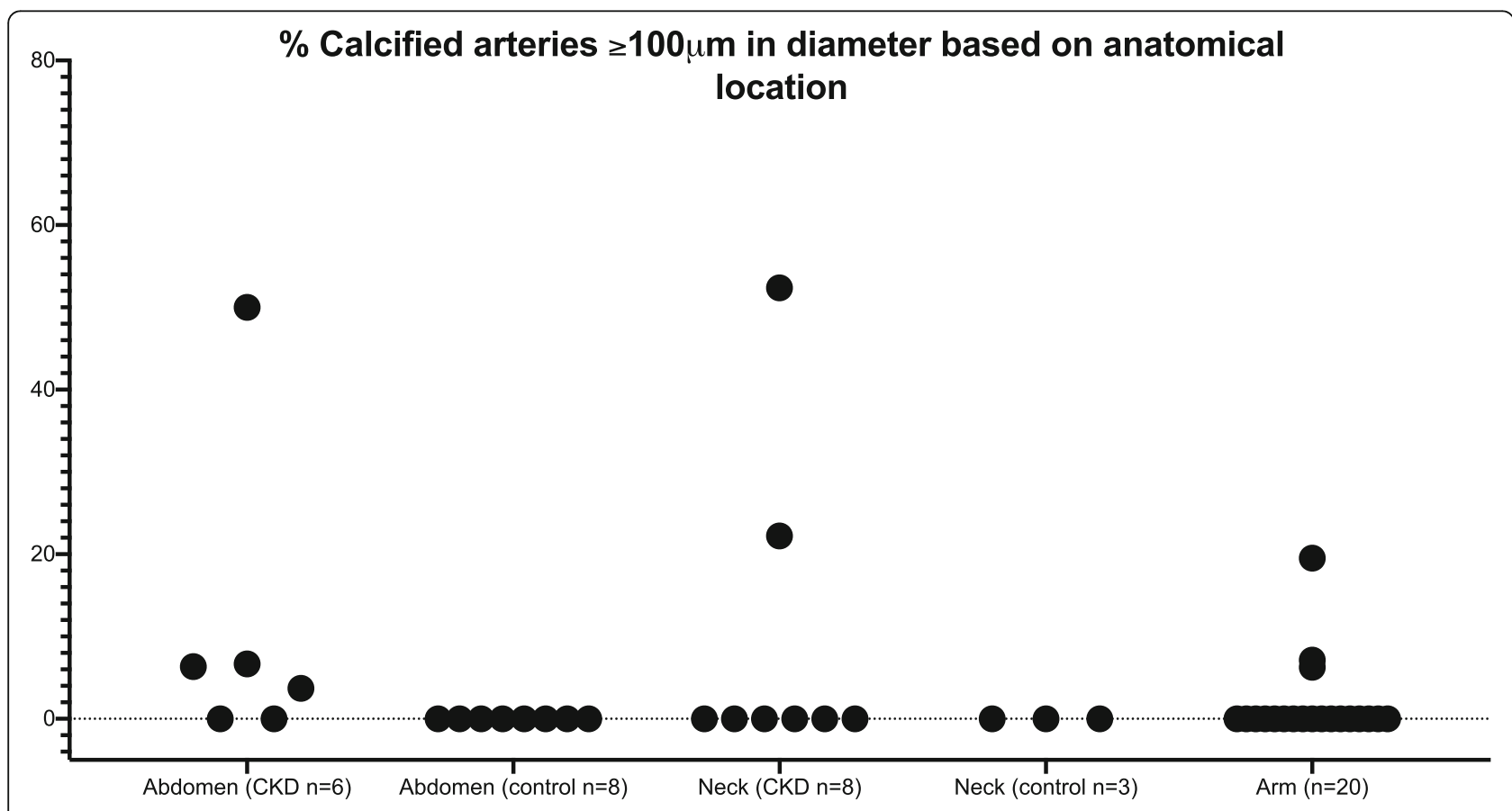

Fig. 2 Percentage of calcified arteries $\geq 100 \mu \mathrm{m}$ in diameter based on anatomical location. Each dot per column represent individual patient 


\section{\% Calcified vessels based on anatomical location}

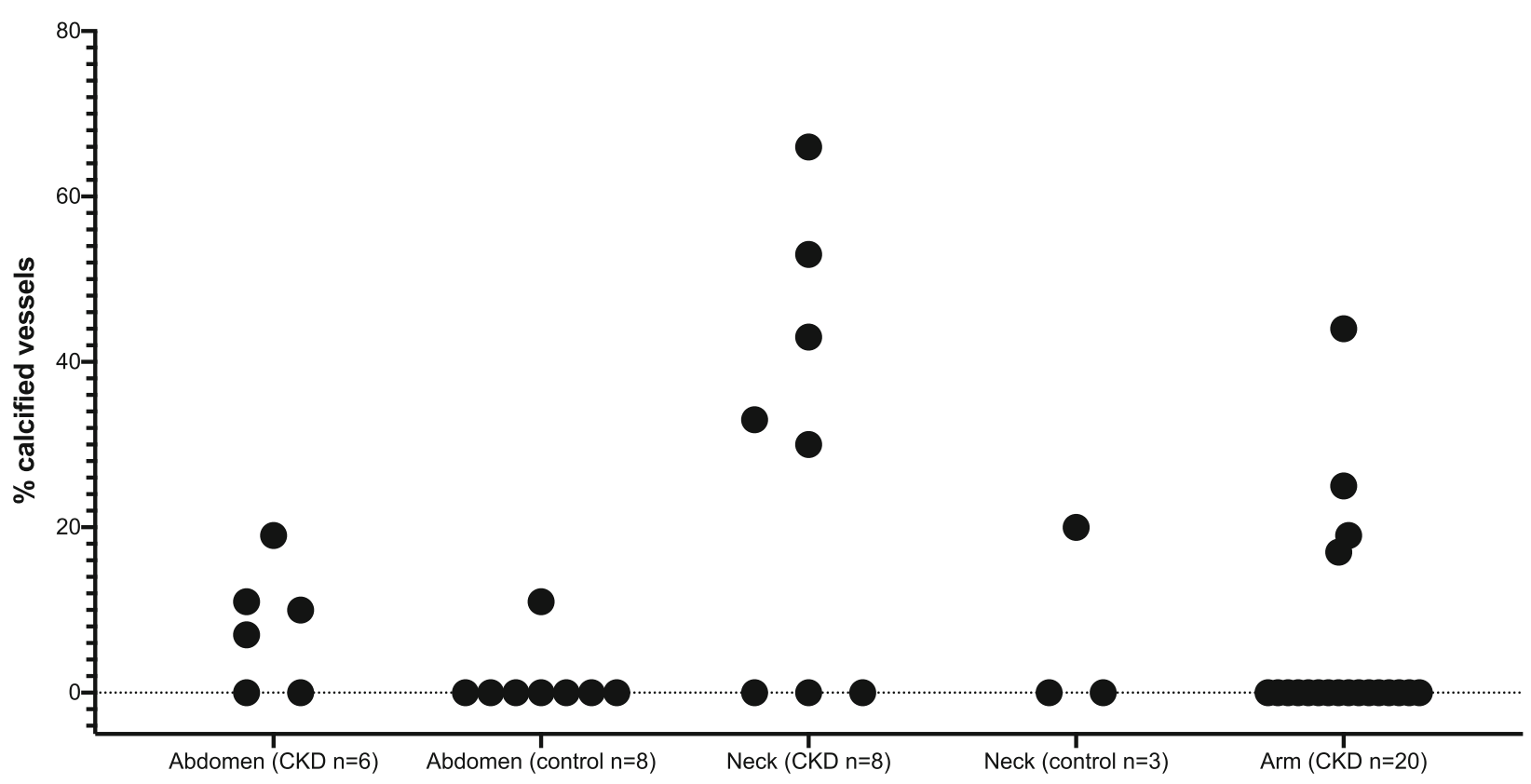

Anatomical location

Fig. 3 Percentage of calcified vessels in each patient based on anatomical location

Median BMI was similar between both groups $(p=0.2)$, as was the co-morbidity profile. With respect to systemic biochemical measures of mineral metabolism and medical therapy, there were no differences in serum calcium, phosphate, ALP or PTH, or in the use of calcium- or non-calcium-based phosphate binders or activated vitamin $\mathrm{D}$ between groups.

\section{No evidence of upregulation of pro-osteogenic gene expression in patients with ESKD or with VC}

De novo expression of pro-osteogenic proteins has been widely implicated as a key driver in the pathogenesis of $\mathrm{VC}$ as well as in vascular lesions of patients with calciphylaxis [15]. Accordingly, we looked for changes in expression of two previously identified pro-calcific factors: tissue non-specific alkaline phosphatase (TNAP), which cleaves the mineralisation inhibitor pyrophosphate to phosphate, and RUNX2, a master transcription factor regulating osteogenic gene programs. Thirteen patients with eligible skin samples were evaluated for mRNA expression. Each skin sample was divided into three segments and tested in triplicate. Results are expressed as mean mRNA expression of each sample. There was minimal variability in gene expression across the three segments of tissue per patient sample. There was no upregulation of gene transcripts in samples with histological evidence of calcification $(n=4)$ compared to those without $(n=9)$ for either TNAP or RUNX2, $p=$
0.76 and $p=0.35$ respectively (Fig. 5a). When comparing gene expression in patients with CKD/ESKD $(n=11)$ to those with no CKD $(n=2)$, there was also no difference in gene expression for TNAP or RUNX2, $p=0.8$ and $p=0.98$ respectively (Fig. 5 b).

\section{Discussion}

To our knowledge, this is the first study to show evidence of medial VC in subcutaneous and dermal vessels in multiple anatomical locations in patients with advanced CKD and ESKD undergoing elective surgery, without peripheral vascular disease or calciphylaxis. Calcification was present in $38 \%$ of samples from patients with CKD/ESKD, predominantly in the subcutaneous tissue and involving the vessel media as well as the IEL. Perieccrine calcification was identified in $64 \%$ of samples with VC, present mainly in patients with ESKD on dialysis. These microvascular calcifications were not related to biochemical markers of mineral metabolism or changes in pro-calcific gene transcripts.

Von Kossa staining is routinely used to identify tissue calcification [2]. Using this stain, two distinct types of $\mathrm{VC}$ were identified in our skin samples, medial calcification and calcification of the IEL. The medial calcification identified in patients with CKD/ESKD was mostly granular but becoming confluent in some of the affected vessels. Calcification identified in the two control patients was more diffuse and milder in nature, which may 


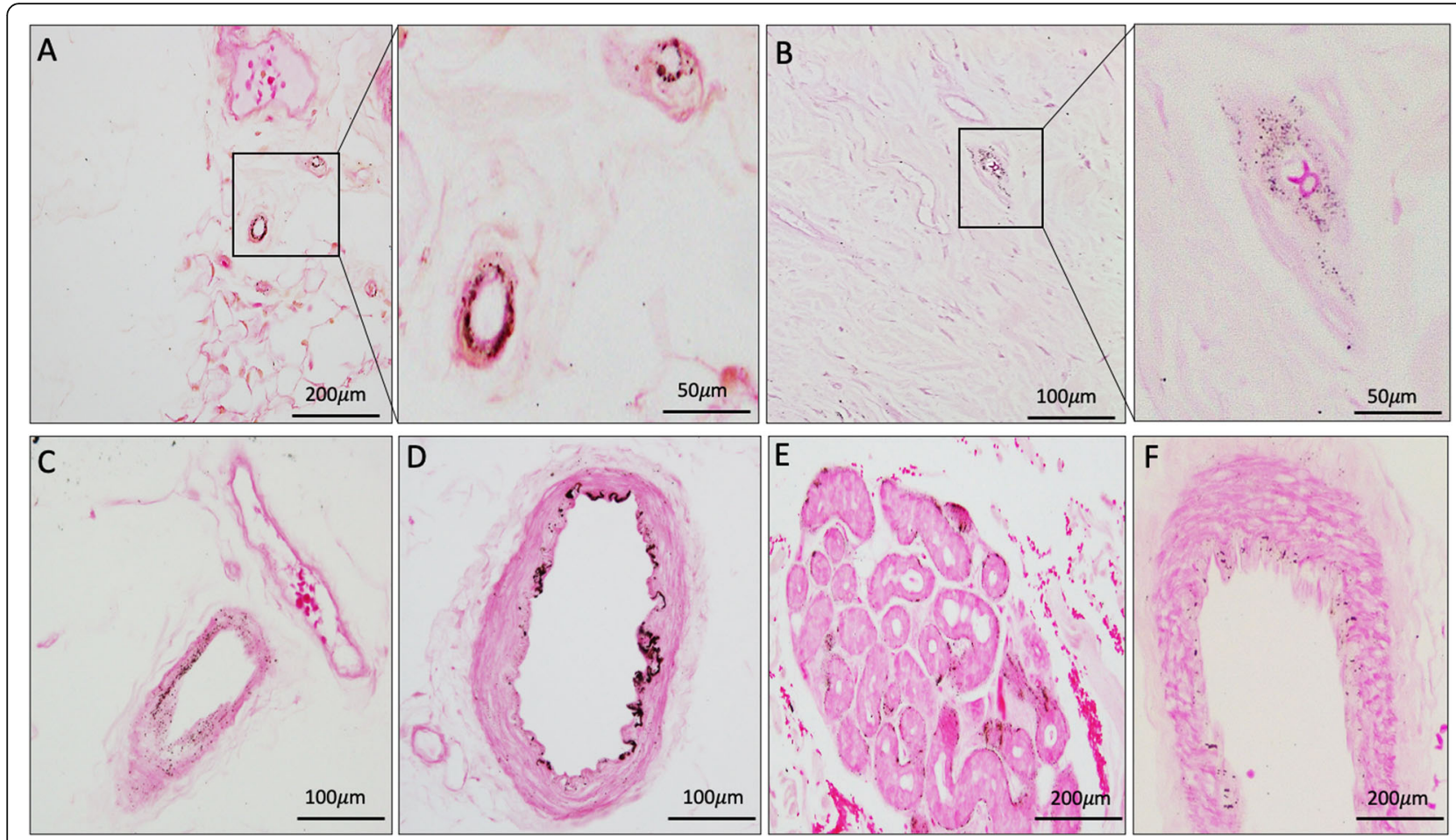

Fig. 4 Micrographs showing various forms of calcification seen in CKD/ESKD (Fig. a-e) and control patient (Fig. f). Von Kossa staining shows (a) Vessels $<50 \mu \mathrm{m}$ in diameter with granular and circumferential medial calcification in subcutaneous tissue and higher magnification insert of vessels highlighted in black box from Panel A. b Vessel $100 \mu \mathrm{m}$ in diameter with granular calcification throughout the media and alongside the internal elastic lamina in subcutaneous tissue and higher magnification of vessel highlighted in black box from Panel B (c) Vessel $100 \mu \mathrm{m}$ in diameter with granular medial calcification in dermal tissue; $\mathbf{d}$ Confluent internal elastic lamina calcification of vessel $>100 \mu \mathrm{m}$ in diameter in subcutaneous tissue; e Perieccrine calcification in dermal tissue; $\mathbf{f}$ Subtle and stippled calcification of vessel $>100 \mu \mathrm{m}$ in diameter in control patient. All photos taken at 20x magnification. Scale bar denoted in each panel

represent a less advanced stage of $\mathrm{VC}$ progression or perhaps a different pathophysiological process. There was no evidence of intimal calcification in our study and there remains conjecture as to whether medial VC and calcification of the IEL represent progression of the same disease process. Multiple studies have shown that arterial medial calcification typically starts along the IEL and in the absence of inflammation [16, 17]. In contrast, arterial intimal calcification is characterized by focal mineral deposition in highly inflamed and necrotic atherosclerotic lesions.

The high prevalence of perieccrine calcification in patients with $\mathrm{VC}$ is a novel finding in our study. There is little literature regarding this histological entity and its clinical significance. Mochel et al. [8] initially described perieccrine calcification in a retrospective cohort of patients with calciphylaxis, suggesting that this histological feature was highly specific to calciphylaxis - the diagnosis of calciphylaxis being made based on this histological feature alone in 4 out of 56 cases. It was also identified in a case report of calciphylaxis [18]. In a recent case series of patients with calciphylaxis (13), perieccrine calcification was not present, however this may have been due to lack of specific staining for calcification. The authors argued that given the subtleness of this histological feature, it may be possible it is routinely missed with standard H\&E staining. In further case series, perieccrine calcification was identified in two biopsies in patients with low to moderate risk of calciphylaxis, and was not significantly prevalent in biopsies of patients with high clinical suspicion of calciphylaxis [7]. The clinical significance of perieccrine calcification remains unknown and it may simply reflect the severity or progression of coexistent VC.

Calcification of skin tissue is considered to be a precursor to the development of calciphylaxis and is a common finding in skin biopsies in patients with this pathology [8]. The specificity of this finding was recently questioned by Ellis and O'Neill in a study of 38 skin biopsies taken for high clinical suspicion for calciphylaxis and compared with lower limb amputation skin samples in patients with ESKD [7]. All previously documented histopathological features commonly associated with calciphylaxis, including medial calcification, intimal thrombosis and extravascular calcification, were prevalent in both high-risk skin lesions and amputation samples. 
Table 3 Differences between CKD/ESKD participants with and without VC

\begin{tabular}{|c|c|c|c|}
\hline Demographic features & VC present $(\boldsymbol{n}=13)$ & VC absent $(\boldsymbol{n}=21)$ & $\boldsymbol{P}$ value \\
\hline Age, years & $57 \pm 13$ & $59 \pm 18$ & 0.60 \\
\hline Sex (male) & $8(61 \%)$ & 17 (80\%) & 0.21 \\
\hline BMI $\left(\mathrm{kg} / \mathrm{m}^{2}\right)$ & $27 \pm 4$ & $29 \pm 6$ & 0.24 \\
\hline Time on dialysis (if ESKD), years & $2.5[1.77-6]$ & $4.2[2-6.5]$ & 0.25 \\
\hline \multicolumn{4}{|l|}{ CKD stage } \\
\hline Pre-dialysis & $1\left(\mathrm{eGFR} 17 \mathrm{ml} / \mathrm{min}^{2}\right)$ & 2 (mean eGFR $20 \pm 0.7 \mathrm{ml} / \mathrm{min}^{2}$ ) & \\
\hline PD & 4 & 1 & \\
\hline $\mathrm{HD}$ & $8(57 \%)$ & $18(86 \%)$ & \\
\hline Diabetes & $4(30 \%)$ & $9(42 \%)$ & 0.55 \\
\hline Hypertension & $10(77 \%)$ & $14(67 \%)$ & 0.52 \\
\hline IHD & $3(23 \%)$ & $4(19 \%)$ & 0.78 \\
\hline PVD & $1(7 \%)$ & $3(14 \%)$ & 0.56 \\
\hline Smoker (current or former) & $8(57 \%)$ & $15(50 \%)$ & 0.66 \\
\hline Previous parathyroidectomy & $2(15 \%)$ & $2(9 \%)$ & 0.64 \\
\hline Calcitriol use & $7(50 \%)$ & $10(34 \%)$ & 0.24 \\
\hline Non-calcium-based binder & $7(50 \%)$ & $11(38 \%)$ & 0.35 \\
\hline Calcium based binder & $2(15 \%)$ & $10(47 \%)$ & 0.06 \\
\hline PTH, pmol/L & 58 [37-184] & 66 [37-123] & 0.41 \\
\hline Total calcium, mmol/L & $2.3 \pm 0.2$ & $2.4 \pm 0.2$ & 0.57 \\
\hline Phosphate, mmol/L & $2 \pm 0.5$ & $1.9 \pm 0.7$ & 0.71 \\
\hline$A L P, I U / L$ & 106 [76-207] & 108 [81-139] & 0.72 \\
\hline Vessel number & $44 \pm 19$ & $39 \pm 15$ & 0.91 \\
\hline Perieccrine calcification & $9(69 \%)$ & $4(19 \%)$ & 0.003 \\
\hline
\end{tabular}

Results are mean \pm SD, median [IQR] or number (\%)

Abbreviations: $A L P$ alkaline phosphatase, $B M I$ body mass index, HD haemodialysis, IHD ischaemic heart disease, $P D$ peritoneal dialysis, $P T H$ parathyroid hormone, PVD peripheral vascular disease

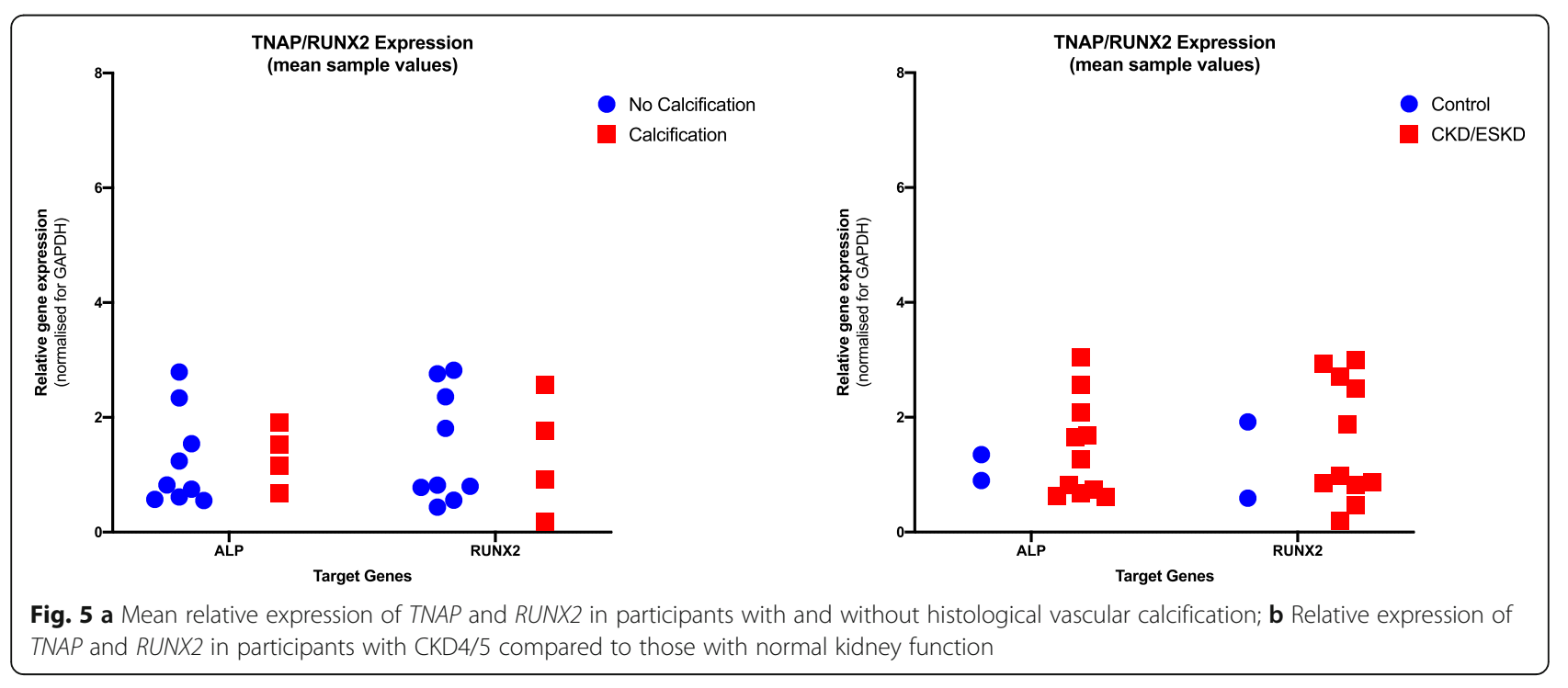


Only the combination of thrombosis and medial calcification was unique to the high-risk skin biopsy group. Our study confirms that medial vascular calcification and perieccrine calcification can be present in "healthy skin" taken from three different anatomical locations, and that these features indeed are not specific to calciphylaxis. We could not identify any evidence of vessel thrombosis, likely due to the low incidence of peripheral vascular disease in our CKD/ESKD cohort. This histological finding may be more prevalent in patients with significant peripheral vascular disease rather than specific to CKD alone.

There were no significant demographic or biochemical differences in patients with CKD/ESKD with and without VC. PTH in patients with VC was similar to those without, but highly variable perhaps reflecting the variability and fluctuations in PTH, as the samples used here only capture one time point. We also identified no significant differences in serum calcium, phosphate or ALP between the two groups. However, the apparent lack of discriminating factors between the two groups may in part reflect the small sample size. It is recognised that medial VC is accelerated in patients with CKD [19]. Schlieper et al identified microcalcifications in the media of iliac arteries in uraemic patients [20] without the presence of intimal calcification or major atherosclerotic plaques. Consistent with our results, the presence of calcification was not associated with abnormalities in serum calcium, phosphate or magnesium, although PTH was not evaluated. Despite these similarities, it is uncertain to what extent $\mathrm{VC}$ in skin and subcutaneous tissue reflects calcification in other vascular beds and whether it is related to more systemic pro-calcific processes affecting the larger central arteries.

RUNX2, a transcription factor, is a master regulator of bone formation and is essential for bone formation and bone remodelling. Some animal studies have shown that RUNX2 expression is required for osteogenic phenotypic change in smooth muscle cells [21]. This transcription factor is also reportedly upregulated in human vascular fragments of large vascular territories where calcification is present [22]. However, evidence of osteogenesis is not a consistent finding across all studies of human VC. We did not see upregulation of RUNX2 or TNAP in the subset of patients tested with histological evidence of calcification. Although osteogenic transformation of vascular smooth muscle cells is one proposed hypothesis for development of medial arterial calcification, other mechanisms have been implicated including elastin degradation, smooth muscle apoptosis and more passive biochemical processes [23]. Lack of osteogenic gene upregulation in our study may also reflect temporal aspects of calcification $[6,24]$ or a different underlying mechanism as a culprit. Human studies involving examination of arterial calcification in breast tissue in patients with CKD demonstrated a universal absence of staining for RUNX2 and osteocalcin in early arterial calcification [6]. A previous study identifying upregulation of osteogenic gene expression in skin was in the setting of calciphylaxis with macroscopic evidence of calcification [15]. This is not reflective of the subtle microscopic calcification identified in our participant cohort that is likely to occur in response to distinct triggers and mechanisms. On the other hand, this may be as a result of technical factors relating to the small sample size, or the sporadic nature of calcification identified on histological examination. Finally, given only a small amount of tissue was processed for PCR, small areas of active calcification may have been missed.

This study was limited by a small sample size and its cross-sectional design with a single skin incisional sample at one time point, without the ability to prospectively follow up VC progression, resolution or patient outcomes. It was not possible to obtain skin samples from control patients to match all anatomical locations and a future case-controlled study may better evaluate the impact of anatomical site on the presence of calcification. Assessment of VC burden at more central sites was also not undertaken. This study had many strengths, however, including a wide distribution of skin anatomical locations sampled as well large incisional biopsy sampling, which allowed for good interpretation of skin architecture and large number of vessels (mean of 40 vessels per slide). Also, patients had no underlying skin pathology to confound results of the study including presence of skin malignancy which is commonly associated with VC.

\section{Conclusion}

In conclusion, this study confirms the presence of medial VC in dermal and subcutaneous tissues in patients with advanced CKD and ESKD. VC was not associated with PTH levels, however, was more likely to be present in patients undergoing parathyroidectomy. The presence of medial $\mathrm{VC}$ and perieccrine calcification is not a specific histological finding for calciphylaxis.

\section{Abbreviations \\ ALP: alkaline phosphatase; CKD: chronic kidney disease; ESKD: end-stage kidney disease; PTH: parathyroid hormone; VC: vascular calcification}

\section{Acknowledgments \\ The authors acknowledge the help of Jayne Amy and the Nephrology Surgical Team at The Royal Melbourne Hospital $(\mathrm{RMH})$ in collecting study samples; and Dr. Moira Finlay, Department of Pathology $\mathrm{RMH}$, for help in reviewing histology}

\section{Authors' contributions}

IR: contributed to the conception, design, data interpretation, critical appraisal of paper and drafting of the work. TH: contributed to the conception, design, data interpretation, critical appraisal of paper and drafting of the work. ES: contributed to the conception, design, data interpretation, critical appraisal of paper and drafting of the work. SH: contributed to critical appraisal of paper and drafting of the work. BW: 
contributed data interpretation. NT: contributed to the conception, design, critical appraisal of paper and drafting of the work. All authors approved final copy of paper.

\section{Funding}

This work was kindly funded by an investigator-initiated research grant from The Royal Melbourne Hospital, Australia. Funding was used for purchase of consumable items for histopathology and RT-PCR investigation.

\section{Availability of data and materials}

Comprehensive data presented in manuscript, tables and figures. Additional data available on request from the corresponding author.

\section{Ethics approval and consent to participate}

Research performed in accordance with the Declaration of Helsinki. The study was approved by the Melbourne Health Human Research Ethics Committee (\#HREC 2017.023). Written informed consent was obtained for all study participants.

\section{Consent for publication}

Not applicable to current manuscript.

\section{Competing interests}

ERS has received research funding from Sanofi, Baxter, and Amgen and holds stock in Calciscon AG, which specializes in performing the $T_{50}$-test. SGH has received research funding, teaching and travel support from AstraZenica, Amgen, Baxter, Sanofi.

NDT has received honoraria, travel support and research funding from Amgen, Shire and Sanofi. TDH, BW and IR declare that they have no competing interests.

Received: 30 January 2020 Accepted: 5 July 2020

Published online: 16 July 2020

\section{References}

1. Ruderman I, Holt SG, Hewitson TD, et al. Current and potential therapeutic strategies for the management of vascular calcification in patients with chronic kidney disease including those on dialysis. Semin Dial. 2018;31(5): 487-99.

2. Smith ER, Hewitson TD, Holt SG. Diagnostic tests for vascular calcification. Adv Chronic Kidney Dis. 2019;26(6):445-63.

3. Goodman WG, Goldin J, Kuizon BD, et al. Coronary-artery calcification in young adults with end-stage renal disease who are undergoing dialysis. N Engl J Med. 2000;342(20):1478-83.

4. London GM, Guerin AP, Marchais SJ, et al. Arterial media calcification in end-stage renal disease: impact on all-cause and cardiovascular mortality. Nephrol Dial Transplant. 2003;18:1731-40.

5. Blacher J, Guerin AP, Pannier B, et al. Arterial calcifications, arterial stiffness, and cardiovascular risk in end-stage renal disease. Hypertension. 2001;38(4): 938-42.

6. O'Neill WC, Adams AL. Breast arterial calcification in chronic kidney disease: absence of smooth muscle apoptosis and osteogenic transdifferentiation. Kidney Int. 2014;85(3):668-76.

7. Ellis CL, O'Neill WC. Questionable specificity of histologic findings in calcific uremic arteriolopathy. Kidney Int. 2018;94(2):390-5.

8. Mochel MC, Arakaki RY, Wang G, et al. Cutaneous calciphylaxis: a retrospective histopathologic evaluation. Am J Dermatopathol. 2013;35(5): 582-6.

9. Essary LR, Wick MR. Cutaneous calciphylaxis. An underrecognized clinicopathologic entity. Am J Clin Pathol. 2000;113(2):280-7.

10. Weenig $\mathrm{RH}$, Sewell LD, Davis MD, et al. Calciphylaxis: natural history, risk factor analysis, and outcome. J Am Acad Dermatol. 2007;56(4):569-79.

11. Brandenburg VM, Sinha S, Specht P, et al. Calcific uraemic arteriolopathy: a rare disease with a potentially high impact on chronic kidney diseasemineral and bone disorder. Pediatr Nephrol. 2014;29(12):2289-98.

12. Nigwekar SU. Calciphylaxis. Curr Opin Nephrol Hypertens. 2017;26(4):276-81.

13. Cerio R, Calonje E. Histopathology of the skin: general principles. Oxford: Wiley-Blackwell; 2010

14. Vinay Kumar RSCSLR, with illustrations by James AP. Robbins basic pathology. Seventh edition. Philadelphia, PA : Saunders, [2003] @2003, Placed Published: 2003
15. Kramann R, Brandenburg VM, Schurgers $L$, et al. Novel insights into osteogenesis and matrix remodelling associated with calcific uraemic arteriolopathy. Nephrol Dial Transplant. 2013;28(4):856-68.

16. Pai $A$, Leaf EM, El-Abbadi $M$, et al. Elastin degradation and vascular smooth muscle cell phenotype change precede cell loss and arterial medial calcification in a uremic mouse model of chronic kidney disease. Am J Pathol. 2011;178(2):764-73.

17. Shanahan CM, Cary NR, Salisbury JR, et al. Medial localization of mineralization-regulating proteins in association with Monckeberg's sclerosis: evidence for smooth muscle cell-mediated vascular calcification. Circulation. 1999;100(21):2168-76.

18. Dookhan C, Ortega LM, Nayer A, et al. Perieccrine and pericapillary calcification in calciphylaxis. J Ren Inj Prev. 2015;4(1):9-10.

19. Gross ML, Meyer HP, Ziebart H, et al. Calcification of coronary intima and media: immunohistochemistry, backscatter imaging, and $x$-ray analysis in renal and nonrenal patients. Clin J Am Soc Nephrol. 2007;2(1):121-34.

20. Schlieper G, Aretz A, Verberckmoes SC, et al. Ultrastructural analysis of vascular calcifications in uremia. J Am Soc Nephrol. 2010;21(4):689-96.

21. Lin ME, Chen T, Leaf EM, et al. Runx2 expression in smooth muscle cells is required for arterial medial calcification in mice. Am J Pathol. 2015;185(7): 1958-69.

22. Donate-Correa J, Martín-Núñez E, Hernández-Carballo C, et al. Fibroblast growth factor 23 expression in human calcified vascular tissues. Aging. 2019;11(18):7899-913.

23. Smith ER. Vascular calcification in uremia: new-age concepts about an oldage problem. Methods Mol Biol. 2016;1397:175-208.

24. Hortells L, Sosa C, Guillen N, et al. Identifying early pathogenic events during vascular calcification in uremic rats. Kidney Int. 2017;92(6):1384-94.

\section{Publisher's Note}

Springer Nature remains neutral with regard to jurisdictional claims in published maps and institutional affiliations.

Ready to submit your research? Choose BMC and benefit from:

- fast, convenient online submission

- thorough peer review by experienced researchers in your field

- rapid publication on acceptance

- support for research data, including large and complex data types

- gold Open Access which fosters wider collaboration and increased citations

- maximum visibility for your research: over $100 \mathrm{M}$ website views per year

At $\mathrm{BMC}$, research is always in progress.

Learn more biomedcentral.com/submissions 